\title{
O ENSINO DA SAÚDE COLETIVA NA UNIVERSIDADE ESTADUAL DE LONDRINA: DA ANÁLISE DOCUMENTAL À PERCEPÇÃO DOS ESTUDANTES
}

\author{
THE TEACHING OF COLLECTIVE HEALTH AT THE LONDRINA STATE UNIVERSITY: \\ FROM DOCUMENT ANALYSIS TO STUDENT PERCEPTION
}

\author{
Flavia Guilherme Gonçalves ${ }^{1}$ \\ Brígida Gimenez Carvalho ${ }^{2}$ \\ Celita Salmaso Trelha ${ }^{3}$
}

\begin{abstract}
Resumo As Diretrizes Curriculares Nacionais para as graduações da área da saúde foram aprovadas em 2002, na tentativa de romper com o paradigma biomédico na produção de conhecimentos e das profissões. Visando a adequar-se àquelas diretrizes, o curso de Fisioterapia da Universidade Estadual de Londrina reformulou sua grade curricular e, dentre as alterações, ampliou a carga horária e reestruturou as disciplinas de Saúde Coletiva. O objetivo deste estudo foi analisar se as ementas de Saúde Coletiva do novo currículo contemplam a formação de competências previstas nas diretrizes curriculares, bem como avaliar a percepção dos estudantes sobre sua formação para atuar na atenção básica. Para isso, procedeu-se à análise documental das ementas e de conteúdo das entrevistas com graduandos do último semestre do curso. Pôde-se constatar que as ementas analisadas contemplam conteúdos essenciais das ciências sociais e humanas na saúde, e que o estágio na atenção básica foi o que mais propiciou a vivência do trabalho em equipe. Os estudantes, apesar de avaliarem positivamente as aulas de Saúde Coletiva, sugeriram que as discussões teóricas fossem mais articuladas ao estágio, pois, desse modo, haveria mais oportunidades de apreender de forma mais efetiva a atuação do fisioterapeuta na atenção básica.
\end{abstract}

Palavras-chave fisioterapia; currículo; atenção básica; ensino superior.
Abstract The National Curricular Directives for undergraduates in the health area were approved in 2002, in an attempt to break the biomedical paradigm with regards to the production of knowledge and professions. With the purpose of adapting to those directives, the Physiotherapy course at the State University of Londrina reformulated its curriculum and, included among the alterations, it expanded the class hours and restructured the Collective Health discipline. The purpose of this study was to analyze whether the Collective Health content of the new curriculum covers the formation of abilities under the curricular directives, as well as evaluating student perceptions with regards to their formation for working with basic health care. Accordingly, a documentary analysis of the content of interviews given by graduating students in the last semester of their courses was carried out. It was ascertained that the content analyzed covers essential aspects of social sciences and humanities in health, and that the apprenticeship in basic health care was what most provided experience in team work. Despite assessing the Collective Health classes positively, the students suggested that the theoretical discussions be more articulated to the apprenticeship, because, accordingly, there would be greater opportunities for learning the work of physiotherapy in the basic health care arena more effectively.

Keywords physiotherapy; curriculum; basic health care; higher education. 


\begin{abstract}
É necessário (...) enfrentar politicamente o 'fetiche da tecnologia' vinculado à concepção de saúde como 'bem de consumo' (e não como um direito), para que se reconheça, sem nenhum desmerecimento aos outros serviços de saúde e especialidades, a urgência de se inverter a proporção de profissionais especializados na atenção básica em relação àqueles dedicados a outros serviços de saúde (Brasil, 2010).
\end{abstract}

O texto constitucional federal define como uma das atribuições do Sistema Único de Saúde (SUS) o ordenamento da formação de recursos humanos para esse setor (Brasil, 1988, art. 200). No entanto, a formação dos profissionais de saúde é reconhecidamente uma área crítica no processo de implementação do sistema de saúde.

A incompatibilidade entre a proposta de reorientação da atenção básica (AB) do SUS - especialmente pautada na Estratégia Saúde da Família, e a grande parte dos recursos humanos que nele atuam - resulta do modelo de ensino que tradicionalmente tem-se baseado na assistência individual, curativa e especializada, em detrimento dos aspectos de promoção e prevenção da saúde coletiva.

Na tentativa de romper com o paradigma biologicista e medicalizante, hospitalocêntrico e procedimento-centrado ainda hegemônico, foram aprovadas em 2002 as Diretrizes Curriculares Nacionais (DCN) (Brasil, 2002) para atender aos novos desafios da contemporaneidade, na produção de conhecimentos e na produção das profissões (Ceccim e Feuerwerkwer, 2004).

A Associação Brasileira de Ensino de Fisioterapia (Abenfisio) em parceria com Organização Pan-Americana de Saúde (Opas) e o Ministério da Saúde (MS) buscam, cada vez mais, sensibilizar e envolver docentes, discentes, gestores e serviços para as questões que abrangem as mudanças nos cursos de graduação. Dentre as atividades propostas por estas instituições, pode ser citada a articulação entre as instâncias formadoras e os serviços de saúde, para produzir movimentos que garantam a inclusão dos estudantes em questões de gestão dos serviços e da participação social por meio de vivências práticas no SUS (Rocha et al., 2008).

Outra atividade proposta foi o estímulo e apoio às instituições formadoras para desencadearem mudanças curriculares com implantação e/ou ampliação de disciplinas de ciências sociais e humanas, entre elas, as de Saúde Coletiva (SC) nas grades curriculares dos cursos de graduação. O fortalecimento desta área nos currículos habilita o futuro profissional a identificar aspectos humanos e sociais envolvidos no processo saúde-doença e a propor ações voltadas à saúde da coletividade (Salmória e Camargo, 2008). 
Visando a adequar-se às novas diretrizes, o curso de Fisioterapia da Universidade Estadual de Londrina (UEL) reformulou seu projeto político pedagógico em 2005, com sua implantação no ano de 2006 (UEL, 2005). Esta mudança buscou assegurar nas diversas disciplinas, tanto nas já existentes como nas que foram inclusas, o equilíbrio do conhecimento nas diferentes áreas e níveis de atenção para garantir uma formação generalista em conformidade com as DCN.

Considerando as mudanças curriculares implantadas no curso de Fisioterapia da UEL, este estudo teve a intenção de analisar se as ementas das disciplinas de SC do novo currículo contemplam a formação de competências previstas nas diretrizes curriculares, bem como a percepção dos estudantes sobre sua formação para atuar na atenção básica.

\section{Metodologia}

Trata-se de uma pesquisa de natureza qualitativa, realizada em duas etapas: a primeira por meio da análise documental dos planos de ensino das disciplinas de SC I, II, III e estágio curricular obrigatório do curso de Fisioterapia da UEL; a segunda por intermédio de entrevistas realizadas com 11 estudantes do último semestre do curso que já haviam cumprido o estágio obrigatório em SC.

A análise dos planos de ensino foi alocada em eixos de acordo com subáreas da Saúde Coletiva referidas pela Associação Brasileira de PósGraduação em Saúde Coletiva: Epidemiologia e Planejamento e Políticas Públicas de Saúde (Abrasco, 2010).

As entrevistas foram gravadas nas dependências do ambulatório de fisioterapia do Hospital Universitário da UEL entre os meses de novembro e dezembro de 2010. Após transcrição, as gravações foram apagadas. Para preservar a identidade dos estudantes, usaram-se letras e números para cada participante: El,..., El1. Os sujeitos foram selecionados por meio de amostragem de conveniência e o número foi determinado pela saturação das respostas (Boni e Quaresma, 2005).

A fim de possibilitar um agrupamento de temas e a categorização do material discursivo (Bardin, 1995), foi elaborado um roteiro semiestruturado com as seguintes questões norteadoras: o que você poderia dizer sobre a atenção básica $(\mathrm{AB})$ dentro do SUS? O que achou da sua formação em SC para atuar na $A B$ ? Quais as contribuições do fisioterapeuta na $A B$ ? Como foi sua formação para o trabalho em equipe inter e multiprofissional?

A pesquisa foi submetida e aprovada pelo Comitê de Ética em Pesquisa da UEL. 


\section{Resultados e discussão}

\section{Análise documental dos planos de ensino das disciplinas de Saúde Coletiva}

O curso de Fisioterapia da UEL foi implantado em 1979 e, desde então, passou por três reformas curriculares, sendo que a atual iniciou-se em 2006. Nessa nova proposta, aumentou-se o número de disciplinas de SC, passando de apenas uma, denominada Fisioterapia Preventiva e Saúde Pública com 136 horas, para quatro disciplinas SC I, II, III e Estágio Curricular Obrigatório, totalizando 382 horas. Esta carga horária está dividida em 120 horas de aulas teóricas e 262 de aulas práticas, conforme o Quadro 1.

\section{Quadro 1}

Série, ementas e carga horária das disciplinas de Saúde Coletiva do curso de Fisioterapia da Universidade Estadua de Londrina, 2010.

\begin{tabular}{|c|c|c|c|c|}
\hline \multirow[t]{2}{*}{ Série } & \multirow[t]{2}{*}{ Disciplinas e ementas } & \multicolumn{3}{|c|}{ Carga horária } \\
\hline & & Teórica & Prática & Total \\
\hline \multirow[t]{4}{*}{$1^{\text {a }}$} & Saúde Coletiva I & 34 & - & 34 \\
\hline & Concepção sobre saúde e doença, organização do serviço & & & \\
\hline & de saúde no Brasil e município, atuação da fisioterapia na & & & \\
\hline & promoção à saúde. & & & \\
\hline \multirow[t]{3}{*}{$2^{\mathrm{a}}$} & Saúde Coletiva II & 17 & 51 & 68 \\
\hline & Território e área de abrangência. Análise das condições de & & & \\
\hline & saúde da população. O papel do fisioterapeuta no serviço. & & & \\
\hline \multirow[t]{3}{*}{$3^{a}$} & Saúde Coletiva III & 17 & 51 & 68 \\
\hline & Domicílio e família. Análise das condições de saúde. & & & \\
\hline & O papel do fisioterapeuta no serviço de saúde. & & & \\
\hline \multirow[t]{4}{*}{$4^{a}$} & Estágio Supervisionado em Fisioterapia em Saúde Coletiva & 52 & 160 & 212 \\
\hline & Desenvolvimento de atividades de aprendizagem & & & \\
\hline & profissional em situações reais de ação do fisioterapeuta & & & \\
\hline & em saúde coletiva. & & & \\
\hline Total & & 120 & 262 & 382 \\
\hline
\end{tabular}

Fonte: Universidade Estadual de Londrina (2005).

Para análise das ementas propostas foi realizado um agrupamento do conteúdo programático em dois eixos: Epidemiologia e Planejamento e Políticas Públicas de Saúde (Tabela 1). 
Distribuição dos conteúdos das disciplinas de Saúde Coletiva por eixos e série no curso de Fisioterapia da Universidade Estadual de Londrina, 2010

\begin{tabular}{|c|c|c|c|c|c|}
\hline Eixos & $1^{\text {a }}$ série & $2^{\mathrm{a}}$ série & $3^{a}$ série & $4^{a}$ série & Total \\
\hline \multicolumn{6}{|l|}{ Epidemiologia } \\
\hline $\begin{array}{l}\text { Análise das condições de saúde da população e } \\
\text { diagnóstico situacional }\end{array}$ & - & $\mathrm{x}$ & $\mathrm{x}$ & $\mathrm{x}$ & 3 \\
\hline Concepção de saúde e doença e fatores determinantes & $\mathrm{x}$ & - & - & $\mathrm{x}$ & 2 \\
\hline Vigilância sanitária e epidemiológica & $\mathrm{x}$ & - & - & - & 1 \\
\hline Epidemiologia & - & $\mathrm{x}$ & $\mathrm{x}$ & - & 2 \\
\hline \multicolumn{6}{|l|}{ Planejamento e Políticas Públicas de Saúde } \\
\hline Organização dos serviços de saúde no Brasil e no & $\mathrm{x}$ & - & - & $x$ & 2 \\
\hline \multicolumn{6}{|l|}{ município } \\
\hline Programa Saúde da Família & - & - & $\mathrm{x}$ & $\mathrm{x}$ & 2 \\
\hline Controle social e participação popular & $x$ & - & - & $\mathrm{x}$ & 2 \\
\hline Território e área de abrangência & $\mathrm{x}$ & $x$ & - & $\mathrm{x}$ & 3 \\
\hline Domicílio e família & - & - & $\mathrm{x}$ & $\mathrm{x}$ & 2 \\
\hline Plano de intervenção individual e coletivo & - & $x$ & $\mathrm{x}$ & $\mathrm{x}$ & 3 \\
\hline Trabalho em equipe & - & $\mathrm{x}$ & - & - & 1 \\
\hline
\end{tabular}

Fonte: Universidade Estadual de Londrina (2005).

Dentre os conteúdos propostos nas disciplinas, verificou-se que análise das condições de saúde e diagnóstico situacional, território e área de abrangência e plano de intervenção individual e coletivo foram abordados em três séries do curso. Já os conteúdos relacionados à vigilância sanitária e epidemiológica e trabalho em equipe, em apenas uma série.

Pode-se observar que o eixo de Planejamento e Políticas Públicas de Saúde apresenta um maior detalhamento em termos de conteúdos abordados e maior contato dos estudantes com estes conteúdos durante a graduação, se comparado ao eixo de epidemiologia.

Os dois eixos abordam temas variados demonstrando a diversidade de campos e maneiras de atuação do fisioterapeuta, cumprindo com o objetivo das ementas de cada disciplina. O papel desse profissional na AB também necessita ser discutido de forma ampliada, pois 
Este novo profissional precisa ter um convívio interdisciplinar e estabelecer novas fronteiras do conhecimento, transitar por todos os setores que visem contemplar as relações entre saúde, ambiente e desenvolvimento permeados pela ética e responsabilidade (Viana, 2005, p. 31).

Mesmo com o estabelecimento das DCN em 2002, as quais preconizam que fisioterapeutas tenham uma formação generalista para atuar nos diversos níveis de atenção à saúde, e apesar de várias experiências acadêmicas já terem incluído a $\mathrm{AB}$ como campo de formação, cabe ressaltar que a atuação do fisioterapeuta nessa área é relativamente nova. O projeto de lei n. 4.261/2004 foi umas das primeiras iniciativas para incluir a categoria no Programa Saúde da Família (Silva e Da Ros, 2007). Posteriormente, em 2008, com a aprovação da portaria GM n. 154, que criou o Núcleo de Apoio à Saúde da Família (Brasil, 2008), é que o trabalho do fisioterapeuta na $A B$ foi oficializado.

Embora houvesse espaços reduzidos para atuação na $\mathrm{AB}$, os conteúdos das ementas de SC da UEL já constavam na nova grade curricular desde 2006. O currículo anterior contava com apenas uma disciplina - Fisioterapia Preventiva e Saúde Pública -, que era dada apenas na segunda série do curso. $\mathrm{O}$ atual conta com quatro disciplinas, o que caracteriza um grande avanço no campo da SC e da $\mathrm{AB}$.

\section{Análise das entrevistas com os estudantes}

\section{A atenção básica no SUS}

Ao discorrerem sobre a AB no SUS, os estudantes apontam como pontos positivos: uma proposta interessante que conta com excelentes profissionais dentro das unidades de saúde da família (USF), que trabalham com qualidade e dedicação. Compreendem que, embora haja ações muito curativas centradas na demanda espontânea e na figura do médico, há também preocupação em executar ações de prevenção de doenças e de promoção da saúde da comunidade.

Indicam como fragilidade o desconhecimento da população em relação às possibilidades de desenvolvimento de ações de promoção e prevenção na USF, pois acham que os usuários procuram a unidade preferencialmente para ações curativas. Na opinião dos estudantes, este fato contribui para a manutenção do modelo hegemônico, acarretando o aumento da demanda, que nem sempre consegue ser atendida em suas necessidades. Essa lógica influencia até mesmo a organização das atividades em grupos, as quais são dirigidas para indivíduos que já possuem alguma doença instalada, como grupos de pessoas com hipertensão, diabetes e lombalgias. Apontam, assim, o distanciamento 
entre a prática profissional nas USF e as propostas estabelecidas na Política Nacional de Atenção Básica, bem como a necessidade de envolvimentos de diversas instituições e atores em prol do alcance das mudanças pretendidas.

A articulação entre gestores de saúde e as diversas instituições formadoras, bem como seu compromisso institucional com a proposta da educação permanente são elementos fundamentais para que todas essas mudanças tenham o potencial de acontecer (Brasil, 2003).

Embora haja esse descompasso, relatam que a $\mathrm{AB}$ oferece um bom serviço e que o SUS, em todas as suas instâncias, deveria ser mais valorizado: “O SUS precisa de mais reconhecimento, tanto pelos funcionários que atuam nele, quanto das pessoas que recebem atendimento (E5)".

Foi observado entre os estudantes uma confusão semântica entre SC e $\mathrm{AB}$, pois as usam como se sinônimos fossem. Poucos relacionam SC à atenção ambulatorial e/ou hospitalar.

A SC caracteriza-se por um campo multidisciplinar, interdisciplinar e transdisciplinar que se aproxima "do planejamento, da gestão e avaliação das políticas de saúde, institucionalizadas em programas e serviços do Sistema Único de Saúde" (Luz, 2009, p. 305), compreendendo todos os níveis de atenção. Já a AB é definida pela PNAB como um conjunto de ações de saúde que engloba promoção, prevenção, diagnóstico, tratamento e reabilitação. É desenvolvida por intermédio do exercício de práticas gerenciais e sanitárias, democráticas e participativas, sob a forma de trabalho em equipe, dirigidas a populações de territórios, e é considerada a porta de entrada preferencial do sistema de saúde (Brasil, 2006).

Essa dificuldade de conceituação poderia ser trabalhada promovendose a integração com outras áreas descritas pelas DCN - como conhecimentos fisioterapêuticos e ciências sociais e humanas - não devendo necessariamente ficar a encargo das disciplinas de SC.

Percebeu-se que os estudantes também usam as palavras 'prevenção' e 'promoção' sem muita distinção. Segundo Buss (2003), as ações preventivas têm caráter de impedir o aparecimento de doenças específicas, utilizandose de divulgação de informação científica e de propostas para a adoção de novos hábitos. Já a promoção vai além da prevenção, uma vez que as ações visam à transformação das condições de vida para o aumento da saúde e do bem-estar geral e não se restringem a uma doença específica.

\section{Percepção sobre as disciplinas de Saúde Coletiva}

Os entrevistados relataram que o período de aproximação da USF nos primeiros anos do curso foi muito importante, pois se sentiram mais familiarizados 
com o território e até mesmo com alguns usuários com quem já haviam tido contato em outros momentos, como visitas domiciliares e atividades desenvolvidas em grupos.

As atividades realizadas 'extramuros' da USF antes da quarta série foram significantes para os estudantes, no sentido de vivenciar a atuação do fisioterapeuta em locais como associações de moradores, empresas de transporte coletivo e, principalmente, nas escolas. Em relação a este último local, a fala dos estudantes prolongava-se, explicando detalhadamente à entrevistadora como aconteciam essas atividades com crianças e com os adolescentes.

Segundo Gallo (2005), as experiências 'extramuros', ou seja, aquelas desenvolvidas nos equipamentos sociais existentes no território de abrangência da unidade, são importantes para incentivar a criatividade nos estudantes e ampliar as possibilidades de abordagem integral tanto em nível individual quanto coletivo.

Os entrevistados ressaltam a importância de todas as disciplinas teóricas e práticas para a formação e apontam, especialmente, o conteúdo sobre o território e área de abrangência e o trabalho em equipe, iniciadas na segunda série, como fundamentais para seu processo de ensino/aprendizagem. No entanto, manifestaram que, por vezes, os conteúdos trabalhados em sala de aula foram repetitivos, espaçados e desarticulados das aulas práticas, principalmente no que tange às atividades de promoção e prevenção. Na opinião dos estudantes este fato prejudicou o estágio, provocando insegurança para o desenvolvimento desse tipo de ação: “A gente chega lá pra promover a saúde e daí fica um pouco perdido, é preciso preparo" (E2).

Segundo o Conselho Federal de Fisioterapia e Terapia Ocupacional (Coffito),

O fisioterapeuta precisa entender que vai promover a atenção específica de sua área, mas ao redor estão inseridas outras questões, como doenças endêmicas, por exemplo, tem que conhecer o perfil epidemiológico se deseja agir como agente formador de idéias e ações, enfim como educador e planejador (Coffito, 2000, p. 17).

\section{Percepção sobre a formação para atuação inter e multiprofissional}

Ao serem questionados sobre a atuação em equipe inter e multiprofissional, os entrevistados relataram com satisfação a experiência vivida na USF. Embora não tenham desenvolvido muitas ações conjuntas com outros profissionais, oportunizaram, nos mais diversos espaços da USF, discussões de casos, as quais se deram durante conversas informais e de forma profícua, com agentes comunitários de saúde, auxiliares de enfermagem, enfermeiros, médicos e profissionais de educação física. Esse contato fez com que reconhecessem a importância da troca de saberes e de diferentes olhares 
da equipe para maior resolutividade dos casos, uma das condições necessárias para reorganização da atenção (Brasil, 2006).

Todas as fases educativas implicadas na formação de uma equipe multiprofissional de saúde, seja em regime escolar ou não, são permeadas atualmente pelo discurso da reorganização dos modelos de atenção e das práticas de saúde, sobretudo no âmbito das políticas que configuram o Sistema Único de Saúde (SUS) dos brasileiros (Mello, Moysés e Moysés 2010, p. 683-684).

De acordo com os estudantes, conteúdos sobre trabalho em equipe foram abordados não só pelas disciplinas de SC, mas também pela disciplina de Habilidades. Relatam que é no estágio de SC que vislumbram a possibilidade real dessa atuação, pois, em analogia com a atuação no estágio hospitalar, dizem que, por mais que haja a oportunidade de discussão de casos, não há interação entre os profissionais. “Lá na UBS deu para perceber que dá para trabalhar em equipe" (E2).

Apesar disso, consideram que a disciplina de SC pouco teorizou sobre o trabalho em equipe. Relatam que durante a graduação tiveram uma breve explanação sobre os profissionais que atuavam na USF e suas atribuições, mas não foi enfatizada a atuação integrada com a ESF e do Núcleo de Apoio a Saúde da Família (NASF).

As experiências sobre trabalho em equipe inter e multiprofissional mais lembradas durantes as entrevistas estavam atreladas à participação em atividades extracurriculares, como o Grupo de Estudos sobre o Envelhecimento, Programa de Educação pelo Trabalho para a Saúde (PET-Saúde) e Projeto de Extensão sobre a Saúde do Cadeirante quanto à Prática de Esporte e Inclusão Social. “Dentro da sala de aula tivemos uma noção de como seria trabalhar em equipe, mas o que mais trouxe para nós essa noção foram os estágios não obrigatórios" (E3).

É importante destacar que, ao citarem o trabalho em equipe, limitaram-se a discorrer sobre a discussão de casos e ações em grupos e não mencionaram conteúdos e habilidades abordados, tanto no eixo da Epidemiologia como quanto o do Planejamento e Políticas Públicas, que poderiam ter sido trabalhados em equipe, uma vez que compõem o campo de competência e de responsabilidade de todos os profissionais da saúde. Por campo entendem-se

os saberes e responsabilidades comuns ou confluentes a várias profissões ou especialidades. Todo o saber básico, por exemplo, sobre o processo saúde-doença. Conhecimentos sobre o funcionamento corporal, sobre a relação profissional/paciente, sobre risco epidemiológico e regras gerais de promoção e prevenção (Campos, 2007, p. 249). 


\section{Contribuição do fisioterapeuta na atenção básica}

Os alunos possuem na ementa dos quatro anos do curso a definição e a atribuição do papel do fisioterapeuta na $\mathrm{SC}$ e na $\mathrm{AB}$, enquanto núcleo de competência e responsabilidade. Por núcleo entende-se "o conjunto de saberes e responsabilidades específicos a cada profissão ou especialidade"; seria a aglutinação de conhecimentos e a conformação de um determinado padrão concreto, demarcaria a identidade profissional. “O núcleo marcaria, dessa forma, a diferença entre os membros de uma equipe" (Campos, 2007, p. 248).

Assim, dentre as atribuições do fisioterapeuta na $\mathrm{AB}$ destacadas pelos alunos, aparecem aquelas comuns aos demais profissionais da saúde: promoção, prevenção, reabilitação individual e em grupo e visitas domiciliares que visam a melhorar a qualidade de vida do indivíduo, sua família e comunidade (Brasil, 2006). Além disso, os fisioterapeutas podem atuar nos diversos ciclos de vida dos seres humanos de forma integrada à equipe multiprofissional.

Os entrevistados mencionaram ainda como um dos papéis do fisioterapeuta a realização de atividades na sala de espera, as quais são compatíveis com a Política Nacional de Humanização, que incentiva a utilização dos espaços dos serviços de saúde como ferramenta para facilitar e otimizar o processo de trabalho. Segundo Pimentel (2009, p. 6), a sala de espera é um espaço que favorece "a expressão, a escuta, a troca entre os atores do complexo cenário da saúde, além de ser um espaço de mediação entre os usuários e os profissionais".

Sobre as funções do fisioterapeuta, os alunos ressaltam que esse profissional na $\mathrm{AB}$ atua como 'porta de entrada' dos serviços de fisioterapia, cabendo-lhe, assim, avaliar e realizar o encaminhamento correto para cada caso. Essa constatação reforça o papel da AB como reorganizadora da atenção (Brasil, 2006).

Contudo, as principais atividades da fisioterapia elencadas foram aquelas dirigidas para a prática curativa, como os atendimentos individuais na USF, visitas domiciliares, grupos de agravos específicos - hipertensão, diabetes e alterações posturais. Ou seja, ainda há traços de uma formação permeada pelo modelo tradicional.

\section{Considerações finais}

As disciplinas de Saúde Coletiva implantadas na reforma curricular do curso de Fisioterapia da UEL visam a formação de profissionais com competências para atuar no Sistema Único de Saúde.

Para os estudantes, a formação teórica dessas disciplinas foi boa, embora repetitiva; as aulas práticas, por sua vez, foram insuficientes para a assimi- 
lação dos objetivos propostos nas ementas. Essa relação soa como incoerente, já que as aulas práticas ultrapassam em 142 horas as aulas teóricas. No entanto, essa incoerência pode ser explicada pelo fato de os estagiários não terem mencionado conteúdos referentes ao eixo da Epidemiologia e de Planejamento e Políticas Públicas.

A formação do fisioterapeuta para atuar em equipe inter e multiprofissional ficou mais evidenciada nas atividades extracurriculares do que nas curriculares. Todavia, foram nos estágios da atenção básica que os alunos tiveram o maior contato com a atuação em equipe, quando comparados com os estágios realizados no ambiente ambulatorial e hospitalar.

Desta forma, conclui-se que a área de Saúde Coletiva no curso de Fisioterapia da UEL, apesar de algumas fragilidades no que tange às aulas práticas, possui nas ementas e conteúdos programáticos, aspectos fundamentais para a formação do egresso, contemplando as importantes áreas do conhecimento em Ciências Sociais e Humanas das DCN.

É notável o avanço que as disciplinas de Saúde Coletiva tiveram dentro da última reforma curricular do curso em foco. Porém, neste estudo foi observada a necessidade de uma maior integração entre os conteúdos teóricos e as atividades práticas desenvolvidas nos estágios, a fim de que o egresso possa assumir os espaços da fisioterapia na atenção básica com maior clareza, tendo em vista o leque de possibilidades de atuação necessárias para a reorganização da atenção básica dentro do SUS.

Pode-se perceber que a Saúde Coletiva é destacada dentro de disciplinas específicas direcionadas para a atenção básica, porém, ela não se enquadra apenas nos conteúdos de ciências sociais e humanas e vai além da atenção primária. Assim, de forma ampliada, a Saúde Coletiva poderia ser abordada conjuntamente com outros conteúdos de específicos da profissão, garantindo a formação generalista preconizada pelas DCN nos diversos níveis da atenção.

O recorte feito por este trabalho, com base na análise da grade curricular e na percepção dos alunos sobre sua formação para atuação na atenção básica, não esgota as possibilidades de investigação desta temática. Fica evidente a necessidade de novas análises, a serem realizadas por outros sujeitos (professores ou egressos, por exemplo), que poderiam ser distintas das apresentadas aqui. Entretanto, este estudo pode contribuir para o aprimoramento do currículo dos cursos de graduação em Fisioterapia, uma vez que apresenta sugestões que minimizariam as dificuldades e fragilidades percebidas pelos sujeitos desta pesquisa. 


\section{Contribuições das autoras}

Flavia Guilherme Gonçalves elaborou o estudo bibliográfico, realizou as entrevistas e redigiu parte do texto. Brígida Gimenez Carvalho realizou levantamento bibliográfico, correções e redigiu parte do texto. Celita Salmaso Trelha procedeu ao levantamento bibliográfico e correções do texto.

\section{Notas}

1 Fisioterapeuta do Núcleo de Apoio à Saúde da Família (Nasf) de Londrina, Paraná, Brasil. Mestranda em Saúde Coletiva pela Universidade Estadual de Londrina (UEL). $<$ flaviadafisio@gmail.com>

Correspondência: Rua Paulo Barreto, 92, CEP 86015-710, Londrina, Paraná, Brasil.

2 Professora do Departamento de Saúde Coletiva da Universidade Estadual de Londrina (UEL), Londrina, Paraná, Brasil. Doutoranda do Programa de Gerenciamento em Enfermagem pela Escola de Enfermagem da Universidade de São Paulo (USP). $<$ brigidagimenez@gmail.com>

3 Professora do Departamento de Fisioterapia da Universidade Estadual de Londrina (UEL), Londrina, Paraná, Brasil. Doutora em Ciências da Saúde pela Universidade Estadual de Londrina (UEL). <celita@uel.br>

\section{Referências}

ABRASCO. A Pós-Graduação em Saúde Coletiva no Brasil: subsídios para o debate. Ofício n. 393/2010. Disponível em: <www. abrasco.org.br/UserFiles/File/FCPSC/2010/ ABRASCO_Contribuicoes_PNPG.pdf $>$. Acesso em: 28 de maio 2012.

BARDIN, Laurence (Org.). Análise de conteúdo. Lisboa. Edições 70,1995.

BONI, Valdete, QUARESMA, Silvia. J. Aprendendo a entrevistar: como fazer entrevistas em ciências sociais. Revista Eletrônica dos Pós-Graduandos em Sociologia
Política da UFSC, Florianópolis,v. 2, n. 1, p. 68-80, jan.-jul. 2005.

BRASIL. Congresso Nacional. Constituição da República Federativa do Brasil. Brasília, DF: Senado Federal, 1988.

Lei n 9.394, de 20 de dezembro de 1996. Dispõe sobre as bases e diretrizes da educação nacional. Brasília, DF: Ministério da Educação e Cultura, 1996.

Ministério da Educação. Conselho Nacional de Educação. Câmara de Educação 
Superior. Resolução CNE/CES n. 4, de 19 de fevereiro de 2002. Diário Oficial da União, Brasília, DF, 4 mar. 2002. Seção 1, p. 11.

Ministério da Saúde. Secretaria de Gestão do Trabalho e da Educação na Saúde. Departamento de Gestão da Educação na Saúde. Caminhos para a mudança da formação e desenvolvimento dos profissionais de saúde: diretrizes para a ação política para assegurar educação permanente no SUS. Brasília, DF: 2003.

Ministério da Saúde. Secretaria de Atenção à Saúde. Departamento de Atenção Básica. Política Nacional de Atenção Básica/Ministério da Saúde, Secretaria de Atenção à Saúde, Departamento de Atenção à Saúde. Brasília, DF: 2006.

- Ministério da Saúde. Portaria GM/MS n. 154, de 24 de janeiro de 2008. Cria e estabelece os critérios para credenciamento dos Núcleos de Apoio à Saúde da Família - Nasf. Disponível em: <www.saude. gov.br/dab>. Acesso em: 14 mar. 2011.

. Ministério da Saúde. Secretaria de Atenção à Saúde. Política Nacional de Humanização. Atenção Básica/Ministério da Saúde, Secretaria de Atenção à Saúde, Política Nacional de Humanização. Brasília, DF: 2010.

BUSS, Paulo M. Uma introdução ao conceito de promoção da saúde. In: CZERESNIA, D.; FREITAS, C. M. (Orgs.). Promoção da saúde: conceitos, reflexões, tendências. Rio de Janeiro: Ed. Fiocruz, 2003. p. 15-38.

CAMPOS, Gastão W. S. Subjetividade e administração de pessoal: considerações sobre modos de gerenciar o trabalho em equipes de saúde. In: MERHY, Emerson. E., ONOCKO, Rosana. (Orgs.). Agir em saúde: um desafio para o público. São Paulo: Hucitec, 2007. p. 229-266.

CECCIM, Ricardo B., FEUERWERKER, Laura C. M. Mudança na graduação das profissões de saúde sob o eixo da integralidade. Cadernos de Saúde Pública, Rio de Janeiro, v. 20, n. 5, p. 1.400-1.410, set.-out. 2004.
COFFITO (Conselho Federal de Fisioterapia e Terapia Ocupacional). Saúde pública: omissão ou responsabilidade? Revista Coffito, São Paulo, n. 7 p. 16-17, jun. 2000.

GALLO, D. L. L. A fisioterapia no Programa Saúde da Família: percepções em relação à atuação profissional e formação universitária. Dissertação (Mestrado em Saúde Coletiva) - Londrina, Universidade Estadual de Londrina, 2005.

LUZ, Madel T. Complexidade do campo da saúde coletiva: multidisciplinaridade, interdisciplinaridade e transdisciplinaridade de saberes e práticas - análise sócio-histórica de uma trajetória paradigmática. Saúde e Sociedade, São Paulo, v. 18, n. 2, p. 304-311, 2009.

MELLO, Ana Lúcia. S. F.; MOYSÉS, Simone T.; MOYSÉS, Samuel. J. A universidade promotora de saúde e as mudanças na formação profissional. Interface - Comunicação, Saúde, Educação, Botucatu, v. 14, n. 34, p. 683692, jul.-set. 2010.

PIMENTEL, A. F. A musicoterapia na sala de espera em uma unidade básica de saúde: os usuários rompem o silêncio. Dissertação (Mestrado em Psicossociologia de Comunidades e Ecologia Social) - Rio de Janeiro, Programa Eicos/Universidade Federal do Rio de Janeiro, 2009.

ROCHA, Vera et al. As diretrizes curriculares e as mudanças na formação de profissionais fisioterapeutas. In: FÓRUM NACIONAL DE ENSINO EM FISIOTERAPIA DA ABENFISIO. Conselho Federal de Fisioterapia e Terapia Ocupacional, Revista Coffito, Brasília, Ano IX, n. 26, p. 22-25, 2008.

SALMÓRIA, Jordana G., CAMARGO, Wander A. Uma aproximação dos signos - fisioterapia e saúde - aos aspectos humanos e sociais. Saúde e Sociedade, São Paulo, v. 17, n. 1, p. 73-84, 2008.

SILVA, D. J.; DA ROS, M. A. Inserção de profissionais de fisioterapia na equipe de Saúde da Família e Sistema Único de Saúde: 
desafios na formação. Ciência \& Saúde Coletiva, Rio de Janeiro, v. 12, n. 6, p. 1.673$1.681,2007$.

UNIVERSIDADE ESTADUAL DE LONDRINA. Resolução Cepe n. 350/2005. Reformula o Projeto Político-Pedagógico do Curso de Fisioterapia, a ser implantado a partir do ano letivo de 2006. Disponível em: <www.uel. $\mathrm{br} /$ prograd/?content $=\mathrm{pp} / \mathrm{pp} . \mathrm{html}>$. Acesso em: 28 de maio 2012.
VIANA, S. B. P. Competências dos fisioterapeutas para a atenção básica em saúde da família: avaliação dos professores e egressos da Univali. Dissertação (Mestrado Profissionalizante em Saúde e Gestão do Trabalho). Itajaí. Universidade do Vale do Itajaí, 2005.

Recebido em 29/07/2011

Aprovado em 07/11/2011 